\title{
Course of illness, hippocampal function, and hippocampal volume in major depression
}

\author{
Glenda M. MacQueen*†, Stephanie Campbell, Bruce S. McEwen§, Kathryn Macdonald*, Shigeko Amano, \\ Russell T. Joffe", Claude Nahmias ${ }^{\ddagger}$, and L. Trevor Young*
}

\begin{abstract}
*Mood Disorders Program, ₹Department of Radiology, McMaster University, Hamilton, ON, Canada L8N 3Z5; §Harold and Margaret Milliken Hatch Laboratory of Neuroendocrinology, The Rockefeller University, New York, NY 10021; and "Office of the Dean, University of Medicine and Dentistry, Newark, NJ 07103

Contributed by Bruce S. McEwen, December 10, 2002
\end{abstract}

\begin{abstract}
Studies have examined hippocampal function and volume in depressed subjects, but none have systematically compared nevertreated first-episode patients with those who have had multiple episodes. We sought to compare hippocampal function, as assessed by performance on hippocampal-dependent recollection memory tests, and hippocampal volumes, as measured in a 1.5-T magnetic resonance imager, in depressed subjects experiencing a postpubertal onset of depression. Twenty never-treated depressed subjects in a first episode of depression were compared with matched healthy control subjects. Seventeen depressed subjects with multiple past episodes of depression were also compared with matched healthy controls and to the first-episode patients. Both first- and multiple-episode depressed groups had hippocampal dysfunction apparent on several tests of recollection memory; only depressed subjects with multiple depressive episodes had hippocampal volume reductions. Curve-fitting analysis revealed a significant logarithmic association between illness duration and hippocampal volume. Reductions in hippocampal volume may not antedate illness onset, but volume may decrease at the greatest rate in the early years after illness onset.
\end{abstract}

$\mathbf{T}$ he importance of the hippocampus in the pathophysiology of major depressive disorder (MDD) is supported by a substantial body of evidence from basic and clinical studies. Studies using magnetic resonance imaging (MRI) have reported that hippocampal (HC) volume is reduced in patients with MDD (1). Sheline and colleagues $(2,3)$ reported bilateral $\mathrm{HC}$ volume reductions in women with MDD. Three studies reported that patients with depression have smaller left $\mathrm{HC}$ volumes than control subjects (4-6), with the reductions restricted to men in one study (6); another study found right $\mathrm{HC}$ volume reductions (7). Several investigators have not found reductions in $\mathrm{HC}$ volume in depressed patients (8-10) including those that have assessed combined volumes of the amygdala-HC complex as one (11-14).

Most of the studies that have found reduced $\mathrm{HC}$ volume examined older depressed subjects $(2,3,7)$. Age itself does not predict $\mathrm{HC}$ volume in depressed subjects, but rather it may be predicted by length of illness and other variables associated with past burden of illness (3). Although past illness may be particularly important in accounting for variations in hippocampus size, patients studied to date are heterogeneous with respect to this variable. Sheline et al. (3) reported that past illness predicted $\mathrm{HC}$ volume reduction; others reported that volumetric reductions were greatest in patients with a chronic course and large number of weeks ill than in those who recovered fully with shorter overall illness duration (4). In contrast, one group found that patients with an older age at onset had smaller HC volumes, and number of depressive episodes was unrelated to $\mathrm{HC}$ volume (7). In another study, there was no evidence for an association between $\mathrm{HC}$ volume and number of depressive episodes, weeks in remission, or number of past hospitalizations (5). Thus it has not been established whether patients have $\mathrm{HC}$ abnormalities that antedate depression or whether accrual of depressive episodes results in altered $\mathrm{HC}$ morphology. Although the studies demonstrating an association between duration of illness and $\mathrm{HC}$ volume loss suggest the latter, it remains possible that patients may be predisposed to a more refractory course of illness as the result of abnormal $\mathrm{HC}$ morphology. To date, this critical variable has not been studied in subjects selected for either minimal or extensive past exposure to depressive episodes and when older subjects are excluded from the analysis.

The relation between $\mathrm{HC}$ morphology and function as assessed by tasks that are specific to the $\mathrm{HC}$ such as recollection memory performance has not been examined adequately. HCdependent recollection memory deficits are amongst the most reliably reported neuropsychological changes in patients with MDD (15-19), and these findings provide support for the hypothesis that the hippocampus is abnormal in MDD. An association between HC volume and global cognitive performance in elderly depressed subjects has been reported (14), and one study found an association between left HC gray-matter density and verbal recognition (4). Whether memory deficits occur only in patients with morphological changes is not established.

To address these questions regarding the relation between depressive episodes and $\mathrm{HC}$ function and morphology, measures were obtained from groups of never treated, postpubertal patients in a first episode (FE) of depression and from a sample of relatively young patients with a similar age of onset but a substantial past illness burden. These groups were chosen specifically to assess the effect of course of illness not confounded by age.

\section{Materials and Methods}

Subjects. Twenty subjects ( 7 men and 13 women) with a FE of nonpsychotic, unipolar depression, diagnosed by the Structured Clinical Interview for DSM-IV (SCID; ref. 20) comprised the FE group. No subjects had ever received pharmacological or psychotherapeutic treatment for a psychiatric illness, and all endorsed the current episode as their first of depression. Collaborative history from families and family physicians corroborated the self-reports of the patients. Patients completed the initial assessment including memory testing free of medication. Antidepressant medication was initiated in some cases before scanning. Twenty age-, sex-, and premorbid IQ-matched subjects with no history of psychiatric illness by SCID were included as control subjects. Control subjects had no known first-degree relative with depression.

Seventeen subjects (11 women and 6 men) with confirmed multiple past episodes (MEs) of depression, as determined by

Abbreviations: MDD, major depressive disorder; $\mathrm{HC}$, hippocampal; $\mathrm{FE}$, first episode; SCID, Structured Clinical Interview for DSM-IV; ME, multiple past episode; Ham-D, Hamilton depression rating scale; BDI-II, Beck Depression Inventory II; GAF, Global Assessment of Function Scale; CGI, Clinical Global Impression of Illness Scale; CFQ, Cognitive Failures Questionnaire.

${ }^{\dagger}$ To whom correspondence should be addressed at: Department of Psychiatry and Behav ioral Neurosciences, 4N77A, McMaster University Medical Centre, 1200 Main Street West Hamilton, ON, Canada L8N 3Z5. E-mail: macqueng@mcmaster.ca. 
both patient report and chart history, were included as the group with a high past total burden of illness. Patients had an average of six past episodes of depression and 10 years of illness. All patients in the ME group had received at least a trial of a serotonergic antidepressant with an average of three trials of medication per patient. Several ME patients had multiple antidepressant trials that included serotonergic, tricyclic, and novel agents as well as monoamine oxidase inhibitors. No patients were receiving atypical antipsychotics, lithium, or other mood stabilizers during any phase of the procedure. Four patients in the ME group had received past treatment with electroconvulsive therapy; none had received electroconvulsive therapy in the year before assessment. Seventeen age-, sex-, and premorbid IQmatched individuals with no history of psychiatric illness served as control subjects for this group.

Exclusion criteria for all patients and controls included $(i)$ substance-related disorder within the past 6 months as determined by the SCID; (ii) lifetime history of substance dependence as measured by the SCID; (iii) any anxiety disorder including posttraumatic stress disorder as determined by the SCID; (iv) use of alcohol or illicit psychoactive substance within $48 \mathrm{~h}$ of cognitive testing; $(v)$ untreated medical illness such as uncontrolled diabetes or other endocrine disorders; ( $v i$ ) history of head injury with loss of consciousness; (vii) history of neurological disease; and (viii) treatment with electroconvulsive therapy within 12 months before assessment. All subjects provided written informed consent and completed the questionnaires and memory assessment on the same day. The study was approved by the ethics boards of St. Joseph's Hospital (Ontario, Canada) and Hamilton Health Sciences Corporation (Ontario, Canada).

Diagnostic and Symptom Questionnaires. Subjects received the SCID for diagnostic clarification and to rule out comorbidity. Information from the SCID was used in conjunction with clinical records and family interviews where possible to ascertain number of past depressive episodes. The National Adult Reading Test (21) was administered as an index of premorbid IQ. On the day of cognitive assessment, subjects received the 21-item Hamilton Depression Rating Scale (Ham-D; ref. 22) and the Beck Depression Inventory II (BDI-II; ref. 23); the Global Assessment of Function Scale (GAF; ref. 24) and Clinical Global Impression of Illness Scale (CGI) also were completed.

Memory Assessment. Subjects completed the Cognitive Failures Questionnaire (CFQ; ref. 25), a measure of individual selfperception of memory impairment. High scores on the CFQ indicate a greater degree of perceived memory impairment, and we have shown previously that subjects with a history of depression have elevated CFQ scores that are not a function of current mood state (16). The California Verbal Learning Test was used as a standard neuropsychological tool to assess immediate and delayed verbal memory; deficits on verbal list learning have been shown previously to be associated with MDD (3).

In addition we used a computerized task, the process dissociation task, to examine recollection and habit memory integrity within a single task (26). This task has been recognized as a valid method of examining recollection memory (hippocampusdependent) processes independent of habit memory (hippocampus-independent) within a single paradigm (27). In contrast to list learning and other tasks that can depend heavily on the ability to categorize information or strategies for learning, this task does not access functions that may depend heavily on frontal lobe integrity. This is important, given recent studies reporting dysfunction in frontal lobe-dependent executive tasks in MDD $(28,29)$. The task is sensitive to recollection memory impairment in unipolar subjects across a variety of mood states (16) and uses stimuli that have been studied extensively in nonpsychiatric populations (30). Eighteen stimulus words are paired with two associative responses that occurred with equal frequency in published norms (e.g., door-knobs, door-knock). Stimuli are presented on an IBM-compatible computer monitor using MICROEXPERIMENTAL software (31). Character size is $\approx 3 \times 4$ $\mathrm{mm}$, and subjects sat $\approx 75 \mathrm{~cm}$ from the monitor.

The first phase of the procedure consists of training, during which a habit is created by repeated association of the word pairs. Word pairs are presented every $2 \mathrm{sec}$ as an incomplete pair (door-kno_-), then for $1 \mathrm{sec}$ as the complete pair (door-knobs). Subjects guess completions that are semantically related to the stimulus word. Unknown to the subjects, the word pairs occur with specific frequencies; one pair (e.g., door-knobs) is presented as the correct response in $67 \%$ of trials, whereas the other word pair (e.g., door-knock) is presented as the correct response in $33 \%$ of trials. Word pairs are presented in random order with the exception that no word pair occurred on more than three consecutive trials. The word pairs that are presented at high or low frequency are counterbalanced across subjects.

After this training phase, subjects immediately proceed to a phase consisting of the presentation of 18 successive study-test lists of eight of the word pairs presented during training. Subjects read the word pairs and are told to remember them for a test that will follow. A mathematical distractor task is presented after presentation of the study list. The key test occurs immediately after the mathematical task when incomplete word pairs appear on the screen at the rate of one pair every $3 \mathrm{sec}$ (door-kno_-). Subjects complete the pair with the word on the immediately preceding study list, and are told to guess if they do not remember.

Recollection scores are obtained by subtracting the incongruent trial (when study-list pairs were the same as the lowfrequency pair during training) probability from the congruent trial (where study-list pairs were the same as the high-frequency pair during training) probability. An estimate of habit is obtained by the formula habit = incongruent probability $/ 1-$ recollection (26).

Image Acquisition and Analysis. Images were obtained on a 1.5-T Sigma GE Genesis-based Echo-Speed scanner running version 5.7 software (General Electric Medical Systems, Milwaukee, WI) using a standard $30-\mathrm{cm}$ circularly polarized head coil. Sagittal anatomic images were acquired by using: a 3D/ FSPGR/20 sequence; flip angle, 8; echo delay time in-phase, 3.9 $\mathrm{msec}$; repetition time, $21.2 \mathrm{msec}$; inversion recovery, $300 \mathrm{msec}$; matrix, $512 \times 256$; field of view, $23 \times 17 \mathrm{~cm}$; scan thickness, 1.2 $\mathrm{mm}$ ). Image processing was performed by using the software of the magnetic resonance workstation manufacturer.

Two raters (S.C. and S.A.) measured unilateral HC graymatter volume based on stereological estimation methods that have been used for both magnetic resonance volumetry and microscopy. Interrater reliabilities for right and left $\mathrm{HC}$ were calculated to be 0.83 and 0.87 , respectively. Measurements were taken starting from the optical section lateral extreme of the hippocampus and working medially. On each, the gray-matter region comprising the $\mathrm{HC}$ was outlined manually, and the area within was calculated.

Anatomic Definition of the Hippocampus. The $\mathrm{HC}$ is primarily a gray-matter complex, bordered superiorly by the fornix-fimbria white-matter junction, inferiorly by para-HC gyrus white matter, anteriorly by white matter separating the $\mathrm{HC}$ from the amygdalar nuclei, posteriorly by the lateral ventricle, medially by subarachnoid spaces of various cisterns, and laterally by the white matter of the corona radiata from the temporal gray matter. The indusium griseum, fornix-fimbria white-matter complex, alveus, and white matter of the para-HC gyrus were excluded from the area measured, as were the amygdala proper and the whitematter border, which separates the hippocampus from the 


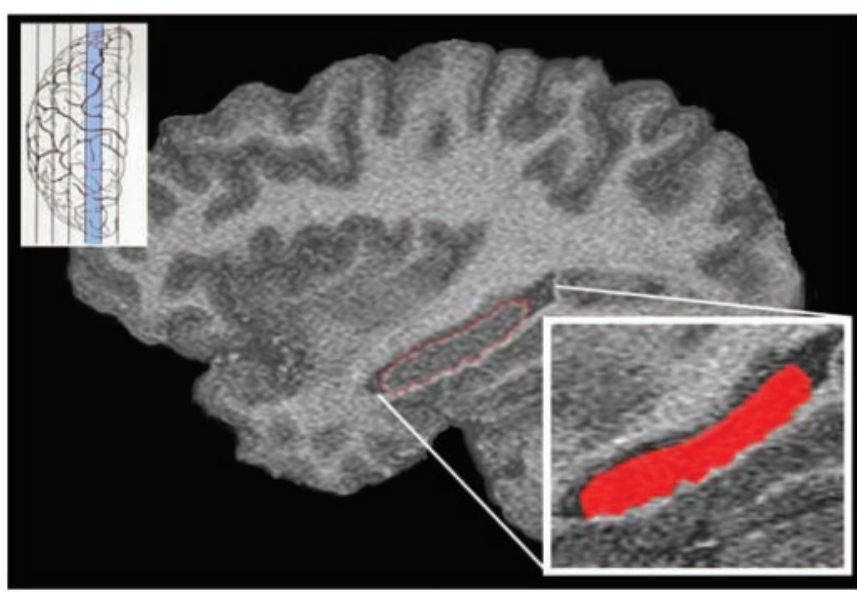

Fig. 1. Saggital MRI of the hippocampus. The HC gray matter (outlined in red) was selected to be measured. Included were the cornu ammonis, dentate gyrus, and subiculum. Excluded were the indusium griseum, amygdalar nuclei, alveus, fimbria, and surrounding white-matter structures.

amygdala. Included were the cornu ammonis and dentate gyrus; because no clear gross anatomic distinction exists between the hippocampus and the subiculum, presubiculum, or parasubiculum, these structures were also included (see Fig. 1)

Anatomic Definition of Total Cerebral Volume. Total cerebral volume included gray and white matter of both hemispheres and the midbrain superior to the pons, with this border used as the demarcation point because it is identified easily.

Statistical Analyses. Absolute left and right $\mathrm{HC}$ volumes as well as volumes corrected for total cerebral volume were examined by using between-within subjects analyses of variance (ANOVAs). The within-group factor included depressed subjects and their age-, sex-, and IQ-matched controls, whereas the between-group factor was FE versus ME patients. This method of analysis was chosen such that these two patient groups could be compared with both their controls and each other within the same analysis. Post hoc analyses were conducted by using the conservative Scheffé test.

Where there was a linear relation between variables, the Pearson correlation was used to determine the significance of relations among volumetric changes, clinical variables, and performance on neuropsychological measures. Curve-fitting analysis that included logarithmic, exponential, power, and quadratic functions was conducted to assess for nonlinear associations between variables. The equation that best described the relation between volumetric and clinical variables was reported if significant.

\section{Results}

Demographic and Clinical Data. Demographic and clinical variables for patient and control groups are summarized in Table 1. FE or multiple-episode patients did not differ from controls on the matched variables of age, sex, and full-scale IQ. One patient in the FE group had well controlled diabetes mellitus, and one in the multiple-episode group had a history of hypothyroidism and was on replacement with normal thyroid indices at the time of assessment. No other patients had a history of significant medical illness. Two patients in each depressed group had remote histories consistent with substance abuse, although not substance dependence, and control subjects were matched for an equal frequency of remote substance abuse. The patient and control groups differed significantly on clinical measures such as Ham-D, BDI-II, CGI, and GAF, but the patient groups did not differ from each other on CGI [df(35), $t=0.98, P=0.33]$, BDI-II $[\mathrm{df}(35), t=1.1, P=0.26]$, or Ham-D [df(35), $t=0.89, P=0.38]$ scores. FE patients had lower GAF scores than ME patients despite equivalent symptom scores on the Ham-D [df(35), $t=$ 2.01, $P=0.05]$. Although $\mathrm{ME}$ patients were older than $\mathrm{FE}$ patients at the time of assessment $[\operatorname{df}(35), t=2.1, P=0.046]$, the patient groups had a similar age of first illness onset [df(35), $t=$ $0.38, P=0.70]$.

Memory Performance. Recollection memory was impaired in both patient groups compared with controls on the process dissociation task [Table 2; $\operatorname{df}(1,35), F=15.9, P<0.001]$. The patient groups did not differ from each other $[\mathrm{df}(1,35), F=1.2, P=$ 0.28]. FE and ME patients performed at the level of control subjects on the measure of habit memory $[\mathrm{df}(1,35), F=0.007$, $P=0.93]$ and showed no differential tendency to guess when compared with control subjects $[\operatorname{df}(1,35), F=0.1, P=0.71]$. There were no associations between Ham-D score at the time of assessment and recollection memory performance $(\mathrm{rsq}=0.001$, $\operatorname{df}(35), F=0.03, P=0.87)$.

There was also a significant difference between patients and controls on verbal memory as assessed by total number of words correct on the California verbal learning test $[\mathrm{df}(1,35), F=7.0$, $P=0.01]$ but no interaction to suggest that $\mathrm{ME}$ patients were relatively worse than controls compared with FE patients [df(1,35), $F=1.9, P=0.17]$.

Patients rated their memory performance as worse than controls on the CFQ [df(1,35), $F=33.4, P<0.001]$. On post hoc analyses, the patient groups did not differ from each other $(P=0.16)$.

Volumetric Data. Patients with multiple episodes of depression had HC volumes that were significantly smaller than both healthy controls and patients in a FE of depression (Figs. 2 and 3). There was a significant interaction between patients and controls [df(1,35), $F=6.4, P=0.016]$. In post hoc analyses, ME patients had smaller left $\mathrm{HC}$ volumes compared with matched controls

Table 1. Demographic and clinical variables of the patients and matched controls

\begin{tabular}{|c|c|c|c|c|}
\hline Variable & FE depression $(n=20)$ & Controls $(n=20)$ & ME depression $(n=17)$ & Controls $(n=17)$ \\
\hline Age, years & $28.4 \pm 11.8$ & $28.4 \pm 11.5$ & $35.9 \pm 11.1$ & $36.2 \pm 11.9$ \\
\hline Sex* & $13 \mathrm{~F} / 7 \mathrm{M}$ & $13 \mathrm{~F} / 7 \mathrm{M}$ & $11 \mathrm{~F} / 6 \mathrm{M}$ & $11 \mathrm{~F} / 6 \mathrm{M}$ \\
\hline FSIQ & $110.6 \pm 6.4$ & $112.7 \pm 6.4$ & $112.7 \pm 6.9$ & $111.7 \pm 5.8$ \\
\hline Ham-D & $19.1 \pm 4.5$ & & $17.5 \pm 6.0$ & \\
\hline BDI-II & $29.7 \pm 10.0$ & & $24.7 \pm 13.7$ & \\
\hline CGI & $3.9 \pm 0.8$ & & $3.6 \pm 0.9$ & \\
\hline GAF & $55.3 \pm 11.4$ & & $62.6 \pm 9.7$ & \\
\hline Age of illness onset, years & $26.3 \pm 12.0$ & & $24.9 \pm 11.6$ & \\
\hline Past depressions & Current episode & & $6.0 \pm 6.0$ & \\
\hline
\end{tabular}

${ }^{*} \mathrm{~F}$, female; $\mathrm{M}$, male. 
Table 2. Hippocampus-dependent recollection memory function, perceived memory impairment (CFQ), and hippocampus volumes

\begin{tabular}{lccc} 
Variable & FE depression $(n=20)$ & Controls $(n=20)$ & ME depression $(n=17)$ \\
\hline $\begin{array}{l}\text { CVLT-total recalled* } \\
\text { Process dissociation }\end{array}$ & $49.3 \pm 14.5$ & $55.3 \pm 13.9$ & $39.2 \pm 16.3$ \\
$\quad$ & & $53.7 \pm 13.6$ \\
$\quad$ Recollection & $0.35 \pm 0.20$ & $0.53 \pm 0.14$ & $0.40 \pm 0.20$ \\
$\quad$ Habit & $0.60 \pm 0.12$ & $0.60 \pm 0.08$ & $0.61 \pm 0.10$ \\
$\quad$ Guessing & $0.65 \pm 0.12$ & $0.66 \pm 0.10$ & $0.63 \pm 0.11$ \\
CFQ & $54.8 \pm 16.0$ & $26.9 \pm 9.6$ & $66.0 \pm 18.0$ \\
Right HC volume, $\mathrm{mm}^{3}$ & $2,793 \pm 303.8$ & $2,784 \pm 342.2$ & $2,392 \pm 256.7$ \\
Left HC volume, $\mathrm{mm}^{3}$ & $2,738 \pm 301.1$ & $2,761 \pm 368.4$ & $2,381 \pm 273.5$ \\
\hline
\end{tabular}

*CVLT, California verbal learning test.

$(P=0.04)$ and FE patients $(P=0.009)$. FE patients did not differ from their matched controls $(P=0.99)$. Right $\mathrm{HC}$ volumes were also decreased only in the ME group, with a similar significant interaction between patient groups compared with controls [df(1,35), $F=7.5, P=0.01]$. In post hoc analyses, ME patients had smaller right $\mathrm{HC}$ volumes than controls $(P=0.04)$, whereas FE patients did not differ from controls $(P=0.99)$. ME patients also had smaller right $\mathrm{HC}$ volumes than FE patients $(P=0.001)$.

Total cerebral volumes did not differ between groups [df(1,35), $F=1.2, P=0.27]$. Consequently, the pattern of results did not change when $\mathrm{HC}$ volumes were examined after correcting for total cerebral volume. Left and right corrected $\mathrm{HC}$ volumes remained smaller when compared with controls in ANOVAs [left: $\operatorname{df}(1,35), F=15.4, P<0.001$; right: $\mathrm{df}(1,35), F=$ $11.8, P=0.002]$; post hoc analyses revealed that ME patients had significantly smaller corrected $\mathrm{HC}$ volumes than controls (left, $P=0.03$; right, $P=0.03$ ). Left or right corrected volumes for $\mathrm{FE}$ groups did not differ when compared with controls (left, $P=$ 0.56 ; right, $P=0.71)$.

In contrast to one recent report of FE subjects (6) but consistent with studies examining depressed subjects with varied illness backgrounds $(7,8,11,14)$, we found no gender by group interaction for $\mathrm{HC}$ volumes in $\mathrm{FE}$ patients [left: $F(1,18)=0.03$, $P=0.86$; right: $F(1,18)=1.54, P=0.23$ ] or ME patients [left: $F(1,15)=0.33, P=0.573$; right: $F(1,15)=0.79, P=0.39]$. When men and women were examined in separate analyses comparing $\mathrm{HC}$ volumes in FE men with controls, there were no differences between gender-specific FE patient groups and healthy controls [men, left HC: $F(1,6)=0.12, P=0.74$; men, right $\mathrm{HC}: F(1,6)=$ $1.11, P=0.33$; women, left $\mathrm{HC}: F(1,12)=0.005, P=0.94$; women, right $\mathrm{HC}: F(1,12)=0.61, P=0.45]$.

Relations Between HC Volumes and Illness Variables. There were trends toward a linear association between length of illness and right and left $\mathrm{HC}$ volumes (right: $r^{2}=-0.29, P=0.08$; left: $r^{2}$ $=-0.29, P=0.08)$. We examined nonlinear equations that might describe the association between length of illness and $\mathrm{HC}$
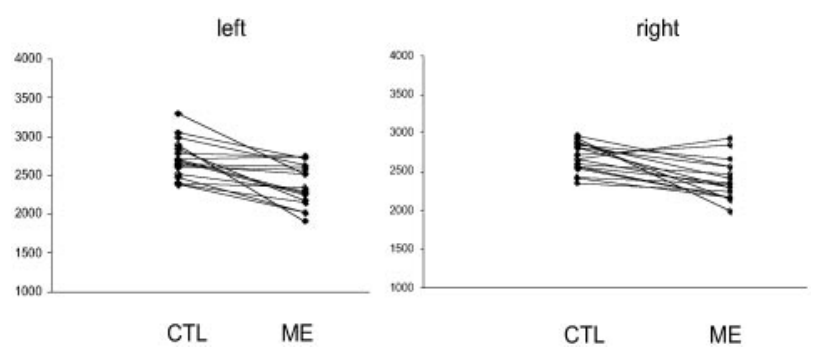

Fig. 2. HC volumes of ME patients and matched control (CTL) subjects. ME patients show significantly smaller $\mathrm{HC}$ volumes (in $\mathrm{mm}^{3}$ ) than age-, sex-, and IQ-matched healthy controls (left $\mathrm{HC}, P=0.04$; right $\mathrm{HC}, P=0.01$ ). volumes, because we have shown previously that the association between illness burden and outcome may be nonlinear (32). In fact, the relation between length of illness and $\mathrm{HC}$ volumes was described best by a logarithmic function that was significant for both the left and right $\mathrm{HC}$ volumes [left HC: rsq $=0.11, \mathrm{df}(35)$, $F=4.4, P=0.04$; right $\mathrm{HC}: \mathrm{rsq}=0.16, \mathrm{df}(35), F=6.8, P=0.03]$ (see Fig. 4). Fig. 4 illustrates the flattening in the curve describing the relation of $\mathrm{HC}$ volumes and length of illness later in the course of illness. Curve-fitting analysis did not reveal any relations between total cerebral volume and duration of illness or number of past episodes

Neither linear nor nonlinear equations described a relation between depressive severity as assessed by the Ham-D or BDI-II and recollection memory function or HC volumes.

\section{Discussion}

This study examines HC function and morphology in nevertreated patients experiencing a FE of depression and in those chosen specifically for multiple past episodes of illness in early and middle adulthood. The results confirm that $\mathrm{HC}$ volume reductions associated with MDD are present in patients with significant past illness and suggest furthermore that such morphological changes are not detectable in adult patients with a FE of depression. Because the oldest patient in the ME group was 51 years old, these changes are not accounted for by increasing age. Both patient groups reported cognitive difficulties and had impairment on list learning and a specific recollection task. A logarithmic function best described the association between illness burden as measured by duration of illness and $\mathrm{HC}$ volumes and suggests that volume reductions occur early in the course of illness but not before first presentation of depression. Taken together, these data highlight the critical interaction between course of illness and $\mathrm{HC}$ pathology in MDD.

The observed decreases in $\mathrm{HC}$ volume might be due to remodeling of key cellular elements involving retraction of dendrites, decreased neurogenesis in the dentate gyrus, and

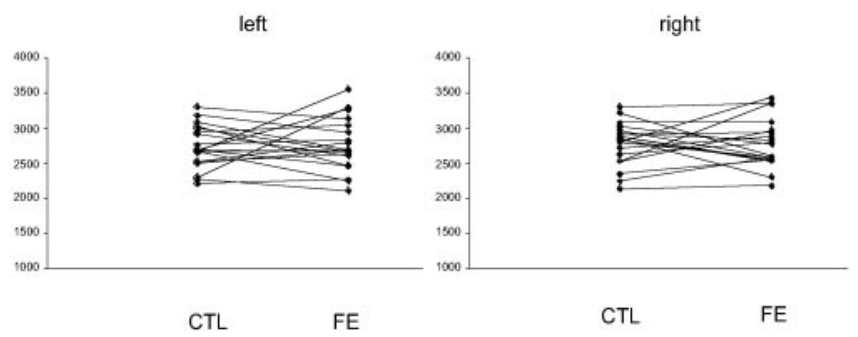

Fig. 3. $\mathrm{HC}$ volumes of FE patients and matched control (CTL) subjects. No significant difference in $\mathrm{HC}$ volume (in $\mathrm{mm}^{3}$ ) is seen between $\mathrm{FE}$ patients and age-, sex-, and IQ-matched healthy controls (left HC, $P=0.99$; right HC, $P=0.99$ ). 



Years since illness onset

Fig. 4. Relation of $\mathrm{HC}$ volumes to length of illness. A logarithmic relation describes the association between duration of illness (in years) and HC volume (in $\mathrm{mm}^{3}$ ) for both left and right $\mathrm{HC}$ volumes.

loss of glial cells (33-38). Potentially reversible remodeling and irreversible cell death are likely caused by dysregulation of glucocorticoid secretion and elevated activity of excitatory amino acid neurotransmitters (39). Indeed, elevated glucocorticoid levels are associated with HC atrophy in rats (39) and primates (40). Patients with MDD have demonstrated abnormalities of the hypothalamic pituitary adrenal (HPA) axis; among the most reproducible findings in patients with MDD is nonsuppression of the HPA axis by dexamethasone, a marker of HPA axis overactivation, along with elevated evening levels of cortisol (41). Because the hippocampus is a major site in the glucocorticoid negative feedback circuit, remodeling or neuronal damage and death may lead to less efficient inhibitory control of the corticotrophin-releasing hormone-producing cells of the hypothalamus, resulting in increased glucocorticoids and worsening of the process (39). A decrease in neurotrophic factors such as brain-derived neurotrophic factor could lead to low $\mathrm{HC}$ volume and vulnerability to subsequent episodes of depression, a result of decreased neurogenesis, increased remodeling of dendrites, and loss of glial cells or increased excitotoxicity $(42,43)$. Repeated exposure to and then withdrawal from antidepressant medication could also contribute to the excitotoxic damage that may underlie the volume reductions in the ME patients. Abrupt withdrawal of the antidepressant imipramine leads to a rapid increase in glutamate activity (44) in animal models, and patients abruptly withdrawn from the serotonergic antidepressant paroxetine have activation of the sympathetic and hypothalamic-pituitary-growth systems (45).

Morphological changes in the hippocampus were not evident in patients in a FE of depression. These data differ somewhat from a recent study in which $\mathrm{HC}$ volume reductions were reported in men but not women in the FE of depression (6). These differences may reflect differences in the age of patients at the time of study. The FE subjects in our study were younger than others studied who had an average age of 40 and included patients up to age $58(6)$. The younger age of onset in our sample is more typical of large-scale epidemiological studies (46); older age at first presentation may be associated with greater exposure to subthreshold depression or to depression associated with vascular or other neurological diseases.

These results have clinical significance in suggesting that morphological changes associated with depression are not present early in illness; consequently, there may be a window of time in which it is possible to arrest or delay progression of the morphological changes associated with MDD. The logarithmic equation that described the association between $\mathrm{HC}$ volumes and illness duration further supports the notion that such intervention is crucial in the early stages of illness. This relation implies a relatively rapid decrease in $\mathrm{HC}$ volumes across the first few years of illness followed by a flattening of the curve, suggesting that after several years of illness, there is, on average, little further decline in $\mathrm{HC}$ volumes. Whether treatment in the early years of illness may ultimately change the clinical course of illness expression and rate of $\mathrm{HC}$ volume loss is not known. The ME patients averaged over three lifetime trials of different antidepressants, and some had many years of treatment with up to a dozen different agents, yet they still displayed the observed morphological and functional abnormalities. This may be because, despite extensive pharmacological treatment histories, they were not treated adequately during the FEs of illness. Alternatively, it is possible that antidepressants, although improving symptoms, do little to prevent the pathophysiological processes associated with chronic, recurrent depression. The long-term benefits of these medications remain to be established, and new agents may need to be developed that interfere with the $\mathrm{HC}$ remodeling that occurs as a consequence of repeated stress (38).

Although morphological changes were not apparent in $\mathrm{FE}$ patients, recollection memory impairment was detectable, which implicates HC dysfunction in these patients and suggests that the dysfunction predates measurable morphological changes. Recollection memory performance was not predicted by Ham-D or BDI-II scores in this study, and using the process dissociation task in a separate group of individuals with current or remitted depression we previously found recollection memory impairment after remission of depressive symptoms (16). These data are consistent with previous work of Sheline et al. (3), which demonstrated list-learning deficits in patients in remission, and other recent reports of memory impairment persisting into the euthymic period $(47,48)$. Whether these deficits ultimately resolve remains to be established. Thus it seems that detectable morphological changes are not required before significant memory abnormalities are detectable, and hippocampus dysfunction, as assessed by recollection memory performance, may be a better marker of the early effects of MDD than structural analyses. The logarithmic association between decreased HC size and length of illness found in this study supports the importance of illness course variables in emergent morphological abnormalities. The fact that the relation between illness duration and $\mathrm{HC}$ volumes was not statistically significant when modeled by a linear curve may explain why other studies that tested only for a linear association could not detect relations between illness burden and $\mathrm{HC}$ volumes. Association between illness burden and $\mathrm{HC}$ volumes could also be obscured if only patients with a long duration of illness are included in the sample, because these patients might be expected to fall only within the flat tail of the curve.

Several factors need to be considered when interpreting the data. First, none of our patients had a prepubertal age of depression onset, nor were they required to have a positive family history of depressive disorder. Selected samples of individuals with childhood-onset depression (49) or strong family histories (50) might represent samples enriched for genetic vulnerability that may be more likely to have abnormal HC morphology at the time of illness onset (51). We focused on patient samples with postpubertal, adolescent, or adult onset of first depression, and these data may not be applicable to all FE patients. Second, it is possible that a greater degree of anatomic resolution would have detected early evidence of morphometric changes in some $\mathrm{HC}$ regions. FE patients and the matched control subjects had right and left $\mathrm{HC}$ volumes that differed by only $\approx 1-2 \%$ in contrast to the $13 \%$ observed in ME patients, however, suggesting that further increasing the sample size would have been unlikely to uncover a real difference between FE patients and their matched controls. Third, the ME patients had variable exposure to antidepressants and benzodiazepines. Although both classes of drugs may have beneficial effects in animal models of depression and chronic stress, respectively, at least in this sample of patients who were currently symptomatic past 
exposure to these medications did not prevent or reverse the appearance of HC damage.

In summary, these data confirm that relatively young adults with multiple episodes of depression have bilateral $\mathrm{HC}$ volume reductions. The association between $\mathrm{HC}$ volume reduction and length of illness was best described by a logarithmic function. Morphological changes were not apparent in patients with a postpubertal FE of depression. Hippocampus dysfunction, as reflected in hippocampus-dependent recollection memory impairment, was apparent in patients with first or multiple episodes of depression and thus seems to affect patients before the

1. Sheline, Y. I. (2000) Biol. Psychiatry 48, 791-800.

2. Sheline, Y. I., Wang, P. W., Gado, M. H., Csernansky, J. G. \& Vannier, M. W. (1996) Proc. Natl. Acad. Sci. USA 93, 3908-3913.

3. Sheline, Y. I., Sanghavi, M., Mintun, M. A. \& Gado, M. H. (1999) J. Neurosci. 19, 5034-5043.

4. Shah, P. J., Ebmeier, K. P., Glabus, M. F. \& Goodwin, G. M. (1998) Br. J. Psychiatry 172, 527-532.

5. Bremner, J. D., Narayan, M., Anderson, E. R., Staib, L. H., Miller, H. L. \& Charney, D. S. (2000) Am. J. Psychiatry 157, 115-117.

6. Frodl, T., Meisenzahl, E. M., Zetzsche, T., Born, C., Groll, C., Jager, M., Leinsinger, G., Bottlender, R., Hahn, K. \& Moller, H. J. (2002) Am. J. Psychiatry 59, 1112-1118.

7. Steffens, D. C., Byrum, C. E., McQuoid, D. R., Greenberg, D. L., Payne, M. E., Blitchington, T. F., MacFall, J. R. \& Krishnan, K. R. (2000) Biol. Psychiatry 48, 301-309.

8. Vakili, K., Pillay, S. S., Lafer, B., Fava, M., Renshaw, P. F., Bonello-Cintron, C. M. \& Yurgelun-Todd, D. A. (2000) Biol. Psychiatry 47, 1087-1090.

9. Rusch, B. D., Abercrombie, H. C., Oakes, T. R., Schaefer, S. M. \& Davidson, R. J. (2001) Biol. Psychiatry 50, 960-964.

10. von Gunten, A., Fox, N. C., Cipolotti, L. \& Ron, M. A. (2000)J. Neuropsychiatry Clin. Neurosci. 12, 493-498.

11. Axelson, D. A., Doraiswamy, P. M., McDonald, W. M., Boyko, O. B., Tupler, L. A., Patterson, L. J., Nemeroff, B., Ellinwood, E. H., Jr., \& Krishnan, K. R. (1993) Psychiatry Res. 47, 163-173.

12. Pantel, J., Schroder, J., Essig, M., Popp, D., Dech, H., Knopp, M. V., Schad, L. R., Eysenbach, K., Backenstrass, M. \& Friedlinger, M. (1997) J. Affect. Disord. 42, 69-83.

13. Coffey, C. E., Wilkinson, W. E., Weiner, R. D., Parashos, I. A., Djang, W. T., Webb, M. C., Figiel, G. S. \& Spritzer, C. E. (1993) Arch. Gen. Psychiatry 50, 7-16.

14. Ashtari, M., Greenwald, B. S., Kramer-Ginsberg, E., Hu, J., Wu, H., Patel, M., Aupperle, P. \& Pollack, S. (1999) Psychol. Med. 29, 629-638.

15. Zakanzis, K. K., Leach, L. \& Kaplan, E. (1998) Neuropsychiatry Neuropsychol. Behav. Neurol. 11, 111-119.

16. MacQueen, G. M., Young, T. L., Marriott, M., Robb, J., Begin, H. \& Joffe R. T. (2002) Acta Psychiatr. Scand. 105, 414-418.

17. Ilsley, J. E., Mofoot, A. P. R. \& O'Carroll, R. E. (1995) J. Affect. Disord. 35, 1-9.

18. Raskin, A., Friedman, A. S. \& DiMascio, A. (1982) Psychopharmacol. Bull. 18, 196-202.

19. Bazin, N., Perruchet, P., De Bonis, M. \& Feline, A. (1994) Psychol. Med. 24, 239-245.

20. First, M. B., Spitzer, R. L., Gibbon, M. \& Williams, J. B. W. (2001) Structured Clinical Interview for DSM-IV-TR Axis I Disorders, Research Version, Nonpatient Edition (Biometrics Res., New York State Psychiatr. Inst., New York).

21. Nelson, H. (1982) National Adult Reading Test Manual (NFER-Nelson, Windsor, ON, Canada).

22. Hamilton, M. (1960) J. Neurol. Neurosurg. Psychiatry 23, 56-72.

23. Beck, A. T. \& Beamesderfer, A. (1974) Mod. Probl. Pharmacopsychiatry 7, 151-169. emergence of significant $\mathrm{HC}$ volume reductions. Treatment early in disease progression may reduce or eliminate the volumetric reductions associated with multiple episodes of illness. Longitudinal studies of patients from early in illness will ultimately be required to evaluate this hypothesis.

We greatly appreciate the assistance of Dr. Michael Marriott, Ms. Helen Begin, and Ms. Cindy D'Amico with data collection and management. G.M.M. is a New Investigator of the Canadian Institutes of Health Research. This work was supported by the Canadian Institutes of Health Research.

24. American Psychiatric Association (1994) Diagnostic and Statistical Manual of Mental Disorders (Am. Psychiatr. Assoc., Washington, DC), 4th Ed.

25. Broadbent, D. E., Cooper, P. F., FitzGerald, P. \& Parkes, K. R. (1982) Br. J. Clin. Psychol. 21, 1-16.

26. Jacoby, L. L. (1998) J. Exp. Psychol. Learn. Mem. Cognit. 24, 3-26.

27. Ruiz-Caballero, J. A. \& Gonzalez, P. (1997) Motiv. Emotion 21, 195-209.

28. Fossati, P. P., Ergis, A. M. \& Allilaire J. F. (2002) Encephale 28, 97-107.

29. Fossati, P. P., Coyette, F., Ergis, A. M. \& Allilaire, J. F. (2002) J. Affect. Disord. 68, 261-271.

30. Jacoby, L. L., Jennings, J. M. \& Hay, J. F. (1996) in Basic and Applied Memory Research: Theory in Context, eds. Herman, D., McEvoy, C., Hertzog, C., Hertel, P. \& Johnson M. K. (Erlbaum, Mahwah, NJ)

31. Schneider, W. (1988) Behav. Res. Methods Instrum. Comput. 20, 206-217.

32. MacQueen, G. M., Young, L. T., Robb, J. C., Marriott, M., Cooke, R. G. \& Joffe, R. T. (2000) Acta Psychiatr. Scand. 101, 374-381.

33. Czeh, B., Michaelis, T., Watanabe, T., Frahm, J., de Biurrun, G., van Kampen, M., Bartolomucci, A. \& Fuchs, E. (2001) Proc. Natl. Acad. Sci. USA 98 12796-12801.

34. Magarinos, A. M., Deslandes, A. \& McEwen, B. S. (1999) Eur. J. Pharmacol. 371, 113-122.

35. Malberg, J. E., Eisch, A. J., Nestler, E. J. \& Duman, R. S. (2000) J. Neurosci. 20, 9104-9110.

36. Rajkowska, G. (2000) Biol. Psychiatry 48, 766-777.

37. Sousa, N., Lukoyanov, N. V., Madeira, M. D., Almeida, O. F. X. \& PaulaBarbosa, M. M. (2000) Neuroscience 97, 253-266.

38. McEwen, B. S. (1999) Annu. Rev. Neurosci. 22, 105-122.

39. Sapolsky, R. M. (2000) Biol. Psychiatry 48, 755-765.

40. Sapolsky, R. M., Uno, H., Rebert, C. S. \& Finch, C. E. (1990) J. Neurosci. 10, 2897-2902.

41. Young, E. A., Haskett, R. F., Grunhaus, L., Pande, A., Weinberg, M., Watson, S. J. \& Akil, H. (1994) Arch. Gen. Psychiatry 51, 701-707.

42. Dowlatshahi, D., MacQueen, G. M. \& Wang, J. F. (1998) Lancet 352, 1754-1755.

43. Chen, B., Dowlatshahi, D. \& MacQueen, G. M. (2001) Biol. Psychiatry 50, 260-261.

44. Harvey, B. H., Jonker, L. P., Brand, L., Heenop, M. \& Stein, D. (2002) Life Sci. 71, 43-54.

45. Michelson, D., Amsterdam, J., Apter, J., Fava, M., Londborg, P., Tamura, R. \& Pagh, L. (2000) Psychoneuroendocrinology 25, 169-177.

46. Wittchen, H. U., Knauper, B. \& Kessler, R. C. (1994) Br. J. Psychiatry Suppl. 26, 16-22.

47. Reischies, F. M. \& Neu, P. (2000) Eur. Arch. Psychiatry Clin. Neurosci. 250, 186-193.

48. Grant, M. M., Thase, M. E. \& Sweeney, J. A. (2001) Biol. Psychiatry 50, 35-43.

49. Steingard, R. J., Renshaw, P. F., Yurgelun-Todd, D., Appelmans, K. E., Lyoo, I. K., Shorrock, K. L., Bucci, J. P., Cesena, M., Abebe, D., Zurakowski, D., et al. (1996) J. Am. Acad. Child Adolesc. Psychiatry 35, 307-311.

50. Drevets, W. C. (2000) Biol. Psychiatry 48, 813-829.

51. Kaufman, J., Martin, A., King, R. A. \& Charney, D. (2001) Biol. Psychiatry 49, 980-1001. 